\section{Ophthalmic statistics note: the perils of dichotomising continuous variables}

\section{INTRODUCTION}

Continuous variables (such as intraocular pressure (IOP), visual acuity, contrast sensitivity) are commonly measured in clinical ophthalmology and vision research. In clinical practice, a 'status' (category) can sometimes be assigned to an individual patient using a cutpoint in the value of a continuous variable; for example, a diagnosis of glaucoma might be confirmed by an elevated IOP measurement (eg, IOP $>21 \mathrm{~mm} \mathrm{Hg}$ ). Indeed much of medicine revolves around an implicit classification of individuals into diseased and non-diseased. In clinical research, continuous variables may likewise be converted to categorical variables, assigning individuals to one of two groups. Although this may be appropriate for some specific studies where the underlying distribution of the variable shows a clear grouping, such dichotomisation has several drawbacks. ${ }^{1}$

Dichotomisation may be driven by the research question, for example, a study to investigate the health service needs of those with low vision, in which dichotomisation uses WHO visual acuity threshold for low vision. ${ }^{2}$ It may sometimes be used to bring the data in line with the clinical classification of patients but often the reason for dichotomisation of data is that it is thought to simplify the statistical analysis (eg, to enable use of a $t$ test or a $\chi^{2}$ test) and the presentation and interpretation of data. However, this simplification has a cost in terms of loss of information ${ }^{3}$ and may compromise the validity of the statistical analysis. We will discuss the disadvantages of dichotomisation and outline some points to consider before categorising continuous data. It should be noted that while the focus here is on categorisation of data into two groups, the problems arising when dichotomising data are inherent with any categorisation of data (two or more groups).

\section{LOSS OF INFORMATION AND STATISTICAL POWER}

Dichotomisation results, first, in the loss of descriptive information on the study population. For example, the nature and extent of differences between individuals with low vision is lost when visual acuity is dichotomised as having/not having low vision. Second, with dichotomisation there is loss of information on between-subject variability in the study population as, for instance, subjects with similar outcome measures but on either side of the threshold will be described and analysed as different while two subjects with values that are on the same side of the threshold, but one near and another a long way from the threshold, will be treated as if they are the same. In addition, it is not possible to quantify linear relationships after dichotomising a variable (eg, it is not possible to quantify the change in $\mathrm{mm} \mathrm{Hg}$ of IOP per $\mathrm{mm} \mathrm{Hg}$ of systolic blood pressure (SBP) increase if IOP has been dichotomised), and any non-linear relationship would be masked by dichotomisation.

There may also be a loss of statistical power (the probability of detecting a true effect of a particular size should it exist) associated with dichotomisation. To maintain statistical power equivalent to that for continuous data, dichotomised data require an increase in sample size. Table 1 shows the sample size required to detect a significant association (correlation) between IOP and SBP, at the 5\% significance level, assuming a linear change of $0.035 \mathrm{~mm} \mathrm{Hg}$ in IOP per mm Hg of $\mathrm{SBP}^{4} 5$ under two scenarios: (a) IOP as a continuous variable (sample size denoted as $n_{\mathrm{o}}$ ) and (b) IOP dichotomised using three different cutpoints (ie, different values for the threshold of IOP that defines the two IOP categories; sample size denoted as $\mathrm{n}_{\mathrm{d}}$ ). Sample size values were calculated using the sample size formulae available for the correlation coefficient ${ }^{6}$ for scenario (a), and for the two-sample $t$ test $^{7}$ (Equation 5.2) for scenario (b). Simulations were generated to calculate $n_{d}$ and the reduction in power for scenario (b) shown in table 1.

When IOP is dichotomised, a larger sample size $\left(n_{d}\right)$ is needed to detect a significant association while maintaining the same power as an analysis with sample size $\mathrm{n}_{\mathrm{o}}$ using IOP as a continuous variable. For example, when IOP is analysed as continuous, the sample size required is 119 individuals for a power of $90 \%$. If IOP is dichotomised using the mean as the cutpoint $(14.5 \mathrm{~mm} \mathrm{Hg})$, then the sample size required to maintain $90 \%$ power increases to 175 individuals -56 additional patients. If the condition of interest is rare, this increase in the required number of patients might render a study infeasible. Alternatively, a reduction in power of at least $15 \%$ would occur if the sample size remains at $\mathrm{n}_{\mathrm{o}}=119$ and IOP was dichotomised.

\section{INTRODUCTION OF BIAS IN ASSOCIATIONS}

In clinical research, the association observed between a risk factor and an outcome can be affected by background factors (such as age) that are associated with the risk factor while also having an influence on the outcome. These background factors are known as confounders. If confounders are present, the estimation of the association of interest between the risk factor and outcome can be biased. Clinical trials are designed to minimise the effect of confounding, with subjects being randomised to intervention or control groups to ensure the groups are balanced with regard to the background factors. However, in epidemiological and other clinical studies, estimates may be biased if the effect of the confounding variable is not properly accounted for in the analysis. If a confounder is taken into account but is dichotomised, this may remove some but not all of the effects of confounding and hence still result in bias. ${ }^{8}$ The magnitude of the bias will depend on the selected cutpoint of dichotomisation and the strength of the confounding effect.

For example, let us investigate if IOP is affected by whether an individual has diabetes. The existing evidence suggests that SBP is related to IOP and also diabetes and as such is a potential confounding variable for the relationship between IOP and whether or not a patient has diabetes (figure 1).

We can fit a linear regression model to estimate IOP with SBP as a continuous covariate and diabetes as a factor with two levels

Table 1 Impact upon power and required sample size due to dichotomisation

\begin{tabular}{|c|c|c|c|c|}
\hline \multirow{2}{*}{$\begin{array}{l}\text { Power to } \\
\text { detect } \\
\text { association } \\
(\%)\end{array}$} & \multirow{2}{*}{$\begin{array}{l}\text { IOP as a } \\
\text { continuous } \\
\text { variable } \\
\mathrm{n}_{\mathbf{o}}\end{array}$} & \multicolumn{3}{|c|}{ IOP as a binary variable } \\
\hline & & $\begin{array}{l}\text { Cutpoint } \\
\text { (mm Hg) }\end{array}$ & $\mathbf{n}_{\mathbf{d}}$ & $\begin{array}{l}\text { Power if } \\
n=n_{0}{ }^{*}(\%)\end{array}$ \\
\hline \multirow[t]{3}{*}{90} & 119 & 14.5 & 175 & 73 \\
\hline & & 16 & 207 & 67 \\
\hline & & 13 & 212 & 67 \\
\hline \multirow[t]{3}{*}{80} & 90 & 14.5 & 133 & 61 \\
\hline & & 16 & 161 & 55 \\
\hline & & 13 & 162 & 54 \\
\hline
\end{tabular}

Assumptions of the model: both IOP and SBP follow a normal distribution with means equal to 14.5 and $135 \mathrm{~mm} \mathrm{Hg}$, respectively, and SDs equal to 2.4 and $20 \mathrm{~mm} \mathrm{Hg}$, respectively.

* Statistical power if sample size $=\mathrm{n}_{\mathrm{o}}$ (as for IOP as a continuous variable) and IOP is dichotomised and analysed accordingly.

IOP, intraocular pressure; SBP, systolic blood pressure. 


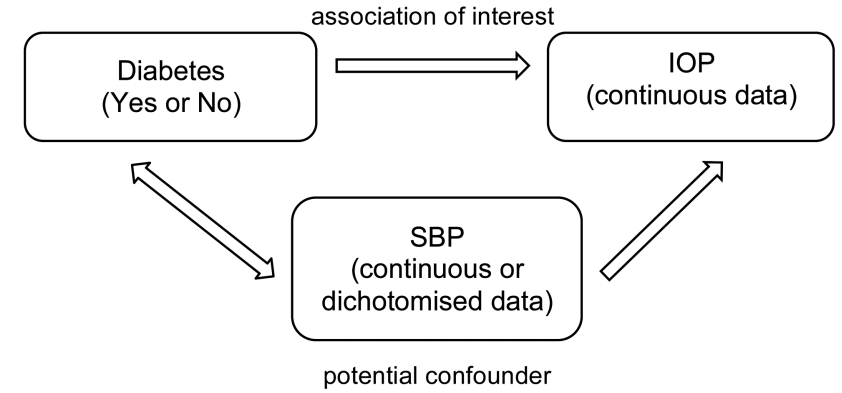

Figure 1 Systolic blood pressure (SBP) as a potential confounder of the relationship between diabetes and intraocular pressure (IOP).

(Yes/No). Let us assume that the true model shows no relationship between IOP and diabetes (ie, a model coefficient of zero for the factor diabetes). The effect of diabetes is correctly estimated as zero using linear regression with SBP as a continuous variable (see dashed lines in figure 2). However, if instead we dichotomise SBP, we find an effect of diabetes on IOP because patients with diabetes are more likely to have SBP above the cutpoint and vice versa (see solid lines in figure 2). In this example, when SBP is dichotomised, data can be fitted using analysis of variance with two factors, diabetes and dichotomised SBP. Figure 2 demonstrates the effect of diabetes adjusted for low and high level of confounding (panels A and B, respectively) dichotomising SBP with a cutpoint of $140 \mathrm{~mm} \mathrm{Hg}$ (median). In figure 2A, we would estimate IOP to be on average $0.13 \mathrm{~mm} \mathrm{Hg}$ higher in the diabetic group compared with the non-diabetic group. With a higher level of confounding (figure 2B), that is, a stronger relationship between SBP and diabetes or larger differences between mean SBP in the diabetes and non-diabetes groups, the bias is even higher $(0.26 \mathrm{~mm} \mathrm{Hg})$. The stronger the confounding between a risk factor and a background variable, the larger the bias introduced by dichotomising the confounding variable. With cutpoint values more extreme than the median, the bias and the probability of concluding that there is a significant effect of diabetes on IOP when there is no true effect (ie, a type 1 error) increase.
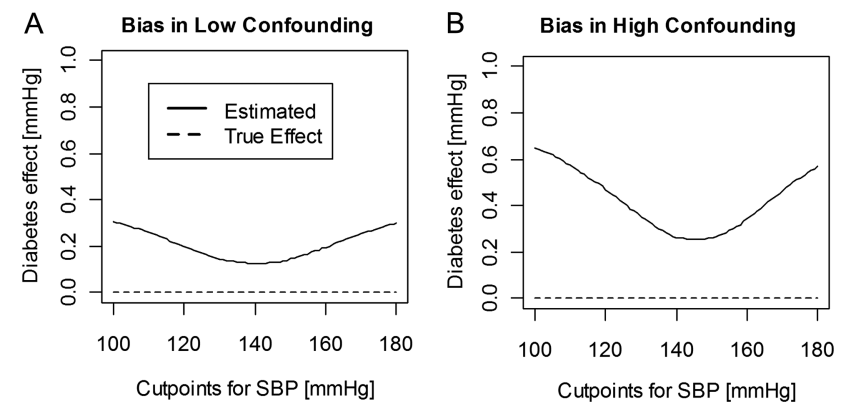

Figure 2 Biased estimates of the effect of diabetes on intraocular pressure (IOP) when confounder systolic blood pressure (SBP) is dichotomised. This simulation assumes that IOP follows a normal distribution and increases on average by $0.035 \mathrm{~mm} \mathrm{Hg}$ per $1 \mathrm{~mm} \mathrm{Hg}$ increase in SBP. We also assume that SBP follows a normal distribution with means of 135 and $145 \mathrm{~mm} \mathrm{Hg}$ for the non-diabetic and diabetic groups, respectively (A) and 135 and $155 \mathrm{~mm} \mathrm{Hg}$ for the non-diabetic and diabetic groups, respectively (B). Finally, we assume that mean IOP is the same in those with and without diabetes. The estimated effect of diabetes on IOP is erroneously positively biased if SBP is dichotomised (see solid curves). To illustrate the average bias, this simulation is based on large number of individuals: 50000 diabetic and 50000 non-diabetic.

\section{POINTS FOR CONSIDERATION}

It is not good practice to power a study, obtain data from a number of individuals and then after completing data collection to underpower the analysis by dichotomisation, thus discarding a substantial amount of the data and information. ${ }^{8}$ It may be appropriate to dichotomise data in certain cases, when the underlying distribution of the variable shows a clear grouping; however, all decisions regarding cutpoints for categorisation should be prespecified before conducting the analysis and reasons for such decisions stated when writing a paper. It is poor practice, for example, to perform the analysis using various data-derived cutpoints and then select the threshold with the most 'significant' result (minimal $\mathrm{p}$ value approach). Using a threshold for dichotomisation that is dependent on the study sample and not the population (eg, when the cutpoint is defined as the sample mean or sample median) will result in a different cutpoint for each study. Thus, results of individual studies will not be generalisable and comparisons between studies may be problematic.

In summary, researchers and clinicians need to be aware of and consider the potential loss of information, decrease in statistical power and the bias that may be introduced by dichotomisation of continuous data.

Phillippa M Cumberland, ${ }^{1}$ Gabriela Czanner, ${ }^{2,3}$ Catey Bunce, ${ }^{4}$ Caroline J Doré, ${ }^{5}$ Nick Freemantle, ${ }^{6}$ Marta García-Fiñana, ${ }^{2}$ On behalf of the Ophthalmic Statistics Group

${ }^{1}$ Centre for Paediatric Epidemiology and Biostatistics, UCL Institute of Child Health, London, UK

${ }^{2}$ Department of Biostatistics, Faculty of Health and Life Sciences, University of Liverpool, Liverpool, UK

${ }^{3}$ Department of Eye and Vision Science, Faculty of Health and Life Sciences, University of Liverpool, Liverpool, UK

${ }^{4}$ NIHR Biomedical Research Centre at Moorfields Eye Hospital NHS Foundation Trust and UCL Institute of Ophthalmology, London, UK

${ }^{5}$ UCL Clinical Trials Unit, University College London, London, UK

${ }^{6}$ Department of Primary Care and Population Health \& PRIMENT Clinical Trials Unit, University College London, London, UK

Correspondence to Phillippa Cumberland, Centre for Paediatric Epidemiology and Biostatistics, University College London (UCL), 30 Guilford Street, London WC1N 1EH, UK; p.cumberland@ucl.ac.uk

Acknowledgements The following members of the Ophthalmic Statistics Group contributed valuable comments and suggestions: Jonathan Cook, Abdel Douiri, Chris Rogers and Irene Stratton.

Collaborators The Ophthalmic Statistics Group.

Contributors PMC, GC and MG-F designed and drafted the paper. CB, CJD and NF conducted an internal peer review of the paper.

Competing interests None of the authors have any conflicts of interest to declare.

Funding PMC is supported by the Ulverscroft Foundation. This work was undertaken at UCL Institute of Child Health/Great Ormond Street Hospital for children, which receives a proportion of funding from the Department of Health's NIHR Biomedical Research Centres funding scheme. Gabriela Czanner and Marta García-Fiñana are grateful to the Clinical Eye Research Centre, St. Paul's Eye Unit, Royal Liverpool and Broadgreen University Hospitals NHS Trust for supporting this work.

Provenance and peer review Commissioned; externally peer reviewed.

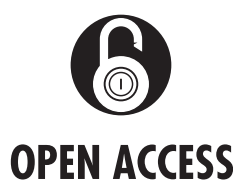

Open Access This is an Open Access article distributed in accordance with the Creative Commons Attribution Non Commercial (CC BY-NC 3.0) license, which permits others to distribute, remix, adapt, build upon this work non-commercially, and license their derivative works on different terms, provided the original work is properly cited and the use is non-commercial. See: http://creativecommons.org/licenses/by-nc/3.0/ 
To cite Cumberland PM, Czanner G, Bunce C, et al. Br J Ophthalmol Published Online First: [please include Day Month Year] doi:10.1136/bjophthalmol-2014304930

Published Online First 28 March 2014

Br J Ophthalmol 2014;98:841-843. doi:10.1136/bjophthalmol-2014-304930

\section{REFERENCES}

1 Altman DG, Royston P. The cost of dichotomising continuous variables. BMJ 2006:332:1080

2 ICD-10 Version 10: 2010. http://apps.who.int/classifications/icd10/browse/2010/en\#/ H53-H54 (accessed 6 Jan 2014).
3 MacCallum RC, Zhang S, Preacher KJ, et al. On the practice of dichotomization of quantitative variables. Psychol Methods 2002;7:19-40.

4 Wong TT, Wong TY, Foster PJ, et al. The relationship of intraocular pressure with age, systolic blood pressure, and central corneal thickness in an Asian population. Invest Ophthalmol Vis Sci 2009:50:4097-102.

5 Memarzadeh F, Ying-Lai M, Azen SP, et al. Associations with intraocular pressure in Latinos: the Los Angeles Latino Eye Study. Am J Ophthalmol 2008:146:69-76.

6 Hsieh FY, Bloch DA, Larsen MD. A simple method of sample size calculation for linear and logistic regression. Stat Med 1998;17:1623-34.

7 Machin D, Campbell MJ, Tan SB, et al. Sample size tables for clinical studies. 3rd edn. Wiley-Blackwell, 2000.

8 Royston P, Altman DG, Sauerbrei W. Dichotomizing continuous predictors in multiple regression: a bad idea. Stat Med 2006;25:127-41. 